\title{
The Vascular Impairment of Cognition Classification Consensus Study
}

\section{Skrobot, Olivia A.}

2017-06

Skrobot, O A , O'Brien , J, Black , S, Chen , C , DeCarli , C , Erkinjuntti , T , Ford , G A , Kalaria , R N , Pantoni , L , Pasquier , F, Roman , G C , Wallin , A , Sachdev , P , Skoog , I , Ben-Shlomo , Y , Passmore , A P , Love , S , Kehoe , P G , VICCCS Grp \& Jokinen , H 2017 , ' The Vascular Impairment of Cognition Classification Consensus Study ' , Alzheimer's \& Dementia , vol. 13 , no. 6 , pp. 624-633 . https://doi.org/10.1016/j.jalz.2016.10.007

http://hdl.handle.net/10138/237009

https://doi.org/10.1016/j.jalz.2016.10.007

publishedVersion

Downloaded from Helda, University of Helsinki institutional repository.

This is an electronic reprint of the original article.

This reprint may differ from the original in pagination and typographic detail.

Please cite the original version. 


\title{
The Vascular Impairment of Cognition Classification Consensus Study
}

\author{
Olivia A. Skrobot ${ }^{\mathrm{a}}$, John O’Brien ${ }^{\mathrm{b}}$, Sandra Black ${ }^{\mathrm{c}}$, Christopher Chen ${ }^{\mathrm{d}}$, Charles DeCarli ${ }^{\mathrm{e}}$, \\ Timo Erkinjuntti ${ }^{\mathrm{f}}$, Gary A. Ford ${ }^{\mathrm{g}}$, Rajesh N. Kalaria ${ }^{\mathrm{h}}$, Leonardo Pantoni ${ }^{\mathrm{i}}$, Florence Pasquier ${ }^{\mathrm{j}}$, \\ Gustavo C. Roman ${ }^{\mathrm{k}}$, Anders Wallin ${ }^{1}$, Perminder Sachdev ${ }^{\mathrm{m}, \mathrm{n}}$, Ingmar Skoog ${ }^{\mathrm{o}}$, VICCCS group ${ }^{1}$, \\ Yoav Ben-Shlomo ${ }^{\mathrm{p}}$, Anthony P. Passmore ${ }^{\mathrm{q}}$, Seth Love ${ }^{\mathrm{a}}$, Patrick G. Kehoe ${ }^{\mathrm{a}, *}$ \\ ${ }^{a}$ Dementia Research Group, Faculty of Health Sciences, School of Clinical Sciences, University of Bristol, Southmead Hospital, Bristol, UK \\ ${ }^{b}$ Department of Psychiatry, University of Cambridge School of Clinical Medicine, Cambridge, UK \\ ${ }^{c}$ Sunnybrook Research Institute, University of Toronto, Canada \\ ${ }^{d}$ Department of Pharmacology, Memory Aging and Cognition Centre, National University of Singapore, Singapore \\ ${ }^{e}$ Alzheimer's Disease Center and Imaging of Dementia and Aging (IDeA) Laboratory, Department of Neurology and Center for Neuroscience, University of \\ California at Davis, Sacramento, CA, USA \\ ${ }^{f}$ Clinical Neurosciences, Neurology, University of Helsinki and Helsinki University Hospital, HUS, Finland \\ ${ }^{g}$ Divison of Medical Sciences, Oxford University, Oxford, UK \\ ${ }^{h}$ Institute of Neuroscience, Newcastle upon Tyne, UK \\ ${ }^{i}$ Department of Neuroscience, University of Florence, Area Drug and Child Health (NEUROFARBA), Florence, Italy \\ ${ }^{j}$ Université Lille, Inserm, CHU Lille, U1171, Degenerative and vascular cognitive disorders, Lille, France \\ ${ }^{k}$ Methodist Neurological Institute, Houston, TX, USA \\ ${ }^{l}$ Institute of Neuroscience and Physiology at Sahlgrenska Academy, University of Gothenburg, Memory Clinic at Department of Neuropsychiatry, Sahlgrenska \\ University Hospital, Mölndal, Sweden \\ ${ }^{m}$ School of Psychiatry, University of New South Wales, Sydney, Australia \\ ${ }^{n}$ CHeBA (Centre for Healthy Brain Ageing), Neuropsychiatric Institute, Prince of Wales Hospital, Randwick, New South Wales, Australia \\ ${ }^{\circ}$ Center for Health and Ageing (AGECAP), Institute of Neuroscience and Physiology, Sahlgrenska Academy, University of Gothenburg, Sweden \\ ${ }^{p}$ School of Social and Community Medicine, University of Bristol, Bristol, UK \\ ${ }^{q}$ Institute of Clinical Sciences, Queens University Belfast, Royal Victoria Hospital, Belfast, UK
}

\begin{abstract}
Introduction: Numerous diagnostic criteria have tried to tackle the variability in clinical manifestations and problematic diagnosis of vascular cognitive impairment (VCI) but none have been universally accepted. These criteria have not been readily comparable, impacting on clinical diagnosis rates and in turn prevalence estimates, research, and treatment.

Methods: The Vascular Impairment of Cognition Classification Consensus Study (VICCCS) involved participants (81\% academic researchers) from 27 countries in an online Delphi consensus study. Participants reviewed previously proposed concepts to develop new guidelines.

Results: VICCCS had a mean of $122(98-153)$ respondents across the study and a $67 \%$ threshold to represent consensus. VICCCS redefined VCI including classification of mild and major forms of VCI and subtypes. It proposes new standardized VCI-associated terminology and future research priorities to address gaps in current knowledge.
\end{abstract}

Discussion: VICCCS proposes a consensus-based updated conceptualization of VCI intended to facilitate standardization in research.

(C) 2016 the Alzheimer's Association. Published by Elsevier Inc. All rights reserved.

Keywords:

Vascular cognitive impairment; Vascular dementia; Guidelines; Criteria; Consensus; Delphi

\footnotetext{
${ }^{1}$ Members are listed at the end of the article.

*Corresponding author. Tel.: +44-117-4147821; Fax: +44-1174147822 .

E-mail address: patrick.kehoe@bristol.ac.uk 


\section{Introduction}

Cerebrovascular pathology, including microinfarcts, lacunar infarcts, larger infarcts (of embolic or thrombotic origin), and white matter lesions, is moderately to strongly associated with cognitive decline [1-4]. Risk factors include hypertension, diabetes mellitus, smoking, atrial fibrillation, positive family history, age, and hypercholesterolemia [5-7], with some risk from APOE ( 84 allele) and MTHFR variants [8]. From the time Hachinski et al. [9] proposed the term multiinfarct dementia, numerous subsequent proposals have tried to capture the clinical and etiologic complexity of cognitive impairment caused by heterogeneous cerebrovascular disease (CVD) and pathologies [10-21]. These include vascular dementia (VaD), vascular cognitive impairment (VCI), subcortical (ischemic) $\mathrm{VaD}$, and vascular cognitive disorder (VCD), which have given rise to multiple criteria and research guidelines $[13,17,19,21]$ that are not readily interchangeable $[22,23]$. These factors contribute to variable prevalence estimates in the literature, as do descriptions of clinical manifestations. However, $\mathrm{VaD}$, used to describe a severe form in the continuum of VCI, is probably the second commonest cause of dementia after Alzheimer's disease (AD), although as populations age this is likely to increase $[13,17,21,24]$. Yet, incidence of dementia is now decreasing in high-income countries, which may partly relate to better CVD management [25]. CVD commonly contributes to many forms of dementia, including AD [26-28], and may be targeted with some success [29], although further research into possible associations and causal relationships is needed. Studies into causes and treatments of AD have greatly outnumbered those for $\mathrm{VaD}$, partly by the availability of widely used diagnostic criteria that continue to evolve [30] and partly because of relatively more funding opportunities.

The lack of consensus criteria for diagnosis of $\mathrm{VaD}$ and VCI has impeded sharing and comparison of data on a larger scale, together with different specialties conducting narrow focused research [31]. Greater harmony of approach within the research community is needed $[23,32]$. A work group convened by the NINDS-CSN made some progress [33], producing detailed research recommendations for VCI. However, their subsequent implementation and adoption remains unclear.

The Vascular Impairment of Cognition Classification Consensus Study (VICCCS) was designed to achieve a broader consensus on the conceptualization of impairment in cognition contributed by vascular pathology, for clinical diagnosis and research. The aim was to provide criteria that could be widely adopted within the field, to underpin future research. VICCCS elaborated previous work to inform the way forward, with input from a broad spectrum of participants from the international research community.

\section{Methodology}

\subsection{Participant selection}

Previous attempts to develop consensus criteria were largely based on comparatively smaller pools of opinion leaders as part of organized meetings, conferences, or symposia [33]. The intention for VICCCS was to draw on the expertise of as many participants from as wide an array of disciplines as possible. Participants for VICCCS were identified through unbiased review of published articles relating to the concept or diagnosis of VaD/VCI in Pubmed, up to August 2010. Several relevant research networks, including the British Association for Stroke Physicians, Alzheimer's Disease Neuroimaging Initiative, and the European Alzheimer's Disease Consortium were also invited.

Nine hundred five individuals were initially identified, although it was not possible to find the contact details of all these most likely because of the fact that some of the source studies were published more than 20 years ago. Further efforts to source these missing contact details were made by inviting all potential participants who were contacted to nominate and provide contact details for potentially interested colleagues. This lead to 789 invitations initially sent that generated a potentially $367(46 \%)$ initially interested pool of international participants. Unlike previous endeavors, VICCCS used periodic internet-based surveys to facilitate greater involvement and promote contributions through providing sufficient time for reflection and responses that were given with anonymity and parity. The study required considerable relevant clinical and research knowledge and time commitment to complete multiple surveys. Nonetheless, on average 122 participants contributed to each round (range 98-153). Of these, a mean of $72 \%$ (range 66\%-76\%) were clinicians with direct involvement in clinical decision-making. The remainder were nonclinical researchers. Average continental distributions were as follows: Europe 63\%, North America 19\%, South America 6\%, Asia 9\%, Africa 2\%, and Australia 1\%. Representation in each round is detailed in Supplementary Table 1. Bar graphs summarizing the professions and affiliations of the authors are also provided in Supplementary Fig. 1. The most common profession was Neurologist (46\%) and the most common affiliation was academic researcher (68\%).

\subsection{VICCCS Delphi process}

We used a Delphi approach, an iterative structured process involving a series of questionnaires with progressive refinement of questions to achieve consensus among respondents [34]. Only the independent moderator (O.A.S., who did not herself participate in the survey) had access to identification details of the respondents. The anonymity of responses facilitated free expression of opinion throughout the study. Structured feedback of responses after each round informed the nature of subsequent questions, allowing unbiased evolution of group judgments that may be difficult face 
to face. A threshold of two-thirds agreement was chosen to represent consensus [35] for issues refined through multiple iterative rounds. Overall, six rounds of web-based surveys were administered, approximately one every 2 months, to maintain engagement. In the first two rounds, opinion was canvassed on published criteria, their utility, and weaknesses. The remaining 4 rounds focused on addressing weaknesses and standardization of terminology. A summary of the topics addressed in each round is provided as Supplementary data.

\section{Results}

\subsection{VICCCS rounds 1 and 2: Critical appraisal of existing proposals}

In the first round, views were sought on the most important issues to be resolved. The extent of use of existing criteria and guidance, identified through literature review, were assessed. We separated questions on "concept" articles, such as those concerning the scope and definitions ( $n=12$ ), from those proposing diagnostic criteria $(n=15)$. Four articles covered both aspects and were included in both sections. Round 1 gathered participants' views on these articles, but also invited additional suggestions for relevant articles that should be considered. Participants were asked to indicate their familiarity with the articles and score their usefulness, from "no longer relevant" to "useful in all cases," and to select three concepts that could form the basis for wider acceptance. To reduce bias in selection that might have been caused by definitions that were older and perhaps more familiar, those selected that scored "useful in most" or useful in all cases were ranked to represent what was a "considered useful vote." The ranking showed that more recently published concepts, even if not widely known, were better regarded as a foundation for future use. The collated scores were fed back to participants in round 2. Participants were then asked to reconsider all articles, including those that might be less familiar, before again ranking the criteria, after which low-ranking criteria would be eliminated from further consideration.

Almost $60 \%$ of respondents ranked the VCI construct of O'Brien et al. [13], representing a broad continuum from mild impairment to dementia, as the preferred conceptual basis. The second and third ranked definitions, which obtained $11 \%$ and $7 \%$ first-preference votes, also encompassed VCI and associated concepts (Supplementary Fig. 2).

In addition, $78 \%$ of respondents felt that the definition of VCI needed to be broader in scope. Therefore, the remaining VICCCS rounds focused on obtaining consensus on a revised conceptual model for VCI. The content of the subsequent rounds was based on responses to early round questions on definition, scope, sensitivity to subtypes of VCI, and clinical utility.

\subsection{Rounds 3-6: Formulation of a revised VCI concept}

In round 3, participants were asked to state their agreement or disagreement with proposed guiding principles for refinement of the concept of VCI. These had more than 94\% agreement; amendments proposed by some participants were reported for comment in round 4 . Consensus guiding principles are listed in Box 1.

Round 3 addressed three areas identified in round 2 as meriting clarification or modification. Although $29 \%$ of respondents thought the O'Brien construct did not need any major improvement, a percentage of respondents felt changes were desirable to its scope (13\%), sensitivity to subtypes (31\%), and descriptiveness (39\%). The subsequent rounds worked toward improving these perceived limitations. Forty-two percent of respondents thought the O'Brien construct was not well aligned with clinical operational criteria. These limitations were subsequently addressed in a focused follow-on Delphi (VICCCS diagnosis) to develop operational criteria (in preparation; however see Box 2 and Supplementary data for some reported findings).

\subsubsection{Scope}

Approximately one-third (34\%) of round 3 participants suggested that other potential mechanisms of VCI should be included in the revised concept. In round 4, participants were asked to vote on inclusion of the suggested mechanisms. There was consensus that the additional mechanisms listed in Table 1 should be included within the revised

Box 1

VICCCS consensus guiding principles of the refinement of the concept of VCI.

1. Be broad and capture forms of vascular- or cerebrovascular-related damage that are likely to contribute to cognitive impairment or dementia.

2. Address shortcomings in both mild and severe forms of VCI and means to assess the transition of patients from one stage to another.

3. Recognize the importance of people who are at risk of VCI; however, their consideration under this construct should be contingent on some level of impairment.

4. Acknowledge that the classification of a patient with a mild form of VCI (i.e., non-dementia) is not necessarily predictive of progression of the impairment to a more severe form of VCI (i.e., dementia).

5. Acknowledge that the classification of a patient with a mild form of VCI (i.e., non-dementia) is not necessarily predictive of an eventual subtype of dementia. 
Box 2

VICCCS proposed definitions of major VCI (VaD) subtypes.

Post-stroke dementia: A patient described as having PSD may or may not have presented evidence of mild cognitive impairment before stroke. The patient may exhibit immediate and/or delayed cognitive decline that begins after, but within 6 months, of stroke, that does not recover. PSD results from different vascular causes and changes in brain. It includes cases with multiple corticosubcortical infarcts, strategic infarcts, subcortical ischemic vascular dementia, and various forms of neurodegenerative pathology, including $\mathrm{AD}$, which develop within 6 months of stroke*. This temporal basis for cognitive decline after stroke differentiates PSD from other forms of major VCI (VaD).

Mixed dementias: A standalone umbrella subgroup termed mixed dementias includes all the phenotypes specified for each combination, that is VCI-AD, VCI-DLB, so forth. It is recommended that a patient is referred to as having "VCI-AD", according to the phenotypes present, rather than less specific mixed dementia, for example. Where discrimination is possible, the order of terms should reflect the relative contribution of the underlying pathology, that is AD-VCI or VCI-AD.

Subcortical ischemic vascular dementia (SIVaD) $:^{\dagger}$ Small-vessel disease is the main vascular cause of SIVaD. Lacunar infarct and ischemic white matter lesions are the main type of brain lesions, which are primarily located subcortically. It incorporates the overlapping clinical entities of Binswanger's disease and the lacunar state.

Multi-infarct dementia (MID): "MID relates to the involvement, and likely contribution, of multiple large cortical infarcts in the development of dementia." The previously mentioned VICCCS definition of PSD is built on the definition of O'Brien et al. [13].

\footnotetext{
* Because a key facet of the definition of PSD is a time component of the appearance of decline within 6 months of having a stroke that does not recover, then irrespective of the presence or absence or comorbid neurodegenerative pathology, the aspect of time should be the primary variable for delineating between PSD (with or without neurodegenerative pathology which if present should be described) and mixed pathology (where the contributing components are described). In other words, PSD and mixed dementias could both have mixed pathology but PSD is recognized by its more acute presentation.

${ }^{\dagger}$ As part of the efforts in VICCCS to standardize the nomenclature and abbreviations to be used in the future, VICCCS diagnosis participants were asked which abbreviation, from those most commonly used for SIVaD, should be taken forward. Initially, no consensus was reached (SIVaD, 36\%; SIVD, 23\%; $\mathrm{SiVaD}, 19 \%$; SiVD, $4 \%$, with $18 \%$ stating no preference) but in the subsequent round, where participants were asked to choose their preference from the two most favored abbreviations from round 4 , most support was for SIVaD (65\%) and therefore adopted.

${ }^{\ddagger}$ Ninety-nine percent of respondents asked about this definition in the VICCCS diagnosis study supported this original definition [11] of SIVaD, whereas 92\% supported it as a diagnostic category. Seventy-six percent of respondents stated that they would use this term clinically.

${ }^{\S}$ Sixty-nine percent of VICCCS diagnosis respondents agreed that MID should be a diagnostic category; however, opinion was split on the use of this term in the clinical setting, with only $52 \%$ in favor of it. There was a consensus (72\%) support for the original definition of MID by Hachinski et al. [9]. MID reflects the traditional view that multiple large cortical infarcts are required for dementia to develop; however, the most frequent objection was use of the word "required." Therefore, to give opportunity for this objection to be considered, a modified definition was also presented along with the original definition for participants to state their support in the subsequent round. The modified definition, as given above, proposed received a consensus support (72\%).
}

concept of VCI. For rounds 4 to 6 , there was also agreement as to what should constitute the arteriopathies subgroup (proposed in the O'Brien construct); however, in VICCCS, specific arteriopathies are a descriptive term of cause rather than a subgroup (Table 2).

\subsubsection{Sensitivity to subtypes}

The O'Brien construct was thought by $31 \%$ of respondents to be limited in capturing subtypes of VCI. Although it acknowledged rare hereditary disorders cause VCI, the construct focused mainly on sporadic forms of VCI. Seventy-eight percent of VICCCS respondents suggested that both hereditary (i.e., "type I" or "familial" VCI) and sporadic (i.e., "type II") should be encompassed within VCI. In round 4 , most (85\%) respondents preferred the terms sporadic and familial to be used as descriptive information for various forms of VCI rather than to define separate categories.

The proposed subtypes of the revised concept of VCI according to the VICCCS are depicted in Fig. 1.

\subsubsection{Mild and major VCI (VaD)}

In the O'Brien construct, $\mathrm{VaD}$ was used as an umbrella term for subgroups of severe forms of VCI. Round 3 partic- ipants were asked whether the term $\mathrm{VaD}$ was still useful. No clear consensus emerged, although a small majority (56\%) favored its continued use. However, the timing of this VICCCS round coincided with the drafting of the fifth edition of Diagnostic and Statistical Manual of Mental Disorders (DSM-5), widely used by clinicians worldwide. The draft DSM-5 proposal was that VaD or major VCDs [36] be shown in parentheses with the description "major neurocognitive impairment because of vascular disease" as a classification group for severe forms of impairment heretofore referred to as $\mathrm{VaD}$ [37]. We therefore sought VICCCS participants" views on the use of the terms "mild" and "major" in relation to VCI. Although only $39 \%$ of round 4 respondents were aware of the draft DSM-5, 71\% agreed that the revised VCI concept should use the terms mild and major to align VICCCS recommendations with DSM-5. In round 5, a $71 \%$ majority supported the terminology "mild forms of VCI" and "major forms of VCI (VaD)."

\subsubsection{Further subtyping of mild forms of VCI}

Subtyping of mild forms of VCI was addressed in rounds 3 to 6 . Most respondents $(68 \%)$ were in favor of specifying subtypes. However, in response to a separate 
Table 1

Clarification of the possible mechanisms of cause of either sporadic or hereditary VCI

\begin{tabular}{ll}
\hline $\begin{array}{l}\text { Mechanisms of cause suggested by VICCCS } \\
\text { participants }\end{array}$ & Percentage support \\
\hline Cerebral amyloid angiopathy & 93 \\
Mixed forms, any neurodegenerative diseases & 93 \\
$\quad$ with CVD (e.g., DLB with CVD) & \\
White matter hyperintensities & 93 \\
Microbleeds/microhemorrhages & 89 \\
Microinfarcts & 89 \\
Arteritis/vasculitis, including both local and & 82 \\
$\quad$ systemic inflammatory syndromes & \\
Subdural or subarachnoid hemorrhage & 70 \\
Option "others" for future developments & 67 \\
Venous thromboses/infarcts & 63 \\
Infectious vasculitis & 53 \\
Hippocampal sclerosis & 42 \\
Angiomatous lesions/vascular tumors with & 33 \\
$\quad$ local steal phenomenon & \\
Chronic migraine & 9
\end{tabular}

Abbreviations: CVD, cerebrovascular disease; DLB, dementia with Lewy bodies; VCI, vascular cognitive impairment; VICCCS, Vascular Impairment of Cognition Classification Consensus Study.

NOTE. Participants were given the opportunity to propose additional causative mechanisms to those previously listed in the O'Brien concept [13]. Percentage support from respondents in the final round is detailed. Those highlighted in italics reached consensus support of $67 \%$ and therefore are recommended.

question $63 \%$ thought that this separation lacked supporting evidence and was premature, and no subtype option could be agreed. Further detail of this is provided in the Supplementary Materials. VICCCS propose that mild $\mathrm{VCI}$ is not subtyped at this time until research provides better justification.

\subsubsection{Further subtyping of major forms of $V C I(V a D)$}

In round 3, respondents were asked to decide which subtypes of dementia proposed by O'Brien et al. should be recognized in VICCCS. Variable levels $(81 \%-50 \%)$ of agreement were found. In round 4 , most respondents (94\%) agreed that this lack of consensus might be overcome if it were possible to avoid mixing site, severity, and mechanism. Ninety-six percent supported an effort to develop a more systematic stepwise approach toward subtyping based on VICCCS proposed categories of Location, Etiology, Domains (affected), and Severity, provisionally named "LEDS" criteria. With this in mind, participants were asked which of the O'Brien subtypes allowed for more mutually exclusive grouping of patients or might be considered better suited as descriptive terms for either the "mechanism" or "location" of damage. The subtypes "specific arteriopathies," "hemorrhagic" and "hypoperfusion" were not supported as standalone subtypes (13\%$18 \%$ ) and thus are recommended as descriptive terms of causal mechanisms in VCI. The remaining subtype terms received variable support between rounds. Round 6 collected a definitive decision, with terms that did not achieve majority (67\%) support to be descriptors. "Subcortical ischemic" (83\%) and "multi-infarct (cortical)" (74\%) were supported as subtypes of major VCI $(\mathrm{VaD})$. As in earlier rounds, poststroke dementia (PSD) was supported (73\%) as a subgroup and $86 \%$ thought it also helpful for clinical diagnosis. In contrast, despite near threshold support (66\%), for consistency "strategic infarct dementia" will also be proposed as a descriptive term for VCI. Additional suggestions for standalone subtypes of VCI were also invited. None of these were supported but "vasculitis"

Table 2

VICCCS-recommended subtypes and descriptive terms

\begin{tabular}{lll}
\hline Subtypes in the VICCCS & Descriptive terms in the VICCCS & O'Brien concept classification and causes of sporadic VCI \\
\hline Poststroke dementia & & Poststroke dementia \\
Multi-infarct (cortical) & & Multi-infarct dementia (cortical VaD) \\
Subcortical ischemic & & Subcortical ischemic VaD \\
& Strategic infarct & Strategic-infarct dementia \\
& Hypoperfusion & Hypoperfusion dementia \\
Mixed dementias & Hemorrhagic & Hemorrhagic dementia \\
Mild VCI & Specific arteriopathies* & Dementia caused by specific arteriopathies \\
& & Mixed AD and VaD \\
& & Vascular mild cognitive impairment
\end{tabular}

Abbreviations: AD, Alzheimer's disease; VaD, vascular dementia; VCI, vascular cognitive impairment; VICCCS, Vascular Impairment of Cognition Classification Consensus Study.

NOTE. Mild VCI, poststroke dementia, multi-infarct (cortical), subcortical ischemic, and mixed dementias are agreed subtypes in the VICCCS. Those in italics fall under the umbrella term major VCI $(\mathrm{VaD})$. Agreed revised definitions of subtypes are detailed in Box 2.

*Specific arteriopathies were agreed in a separate question for two rounds to include genetic, hereditary and developmental anomalies (e.g., Fabry's disease, sickle cell disease, cerebral autosomal-dominant arteriopathy with subcortical infarcts and leukoencephalopathy [CADASIL], and cerebral autosomal recessive arteriopathy with subcortical infarcts and leukoencephalopathy [CARASIL]), small-vessel disease from chronic hypertension and/or diabetes, inflammatory/ immunological vasculitis, Moyamoya disease, and intracranial atherosclerosis.

${ }^{\dagger}$ A revised holistic subtype of mixed dementias was developed and agreed over the course of number of rounds to replace "mixed AD and VaD." VICCCS agreed descriptive terms include strategic infarct, hypoperfusion, hemorrhagic, and specific arteriopathies.

${ }^{\ddagger}$ Vasculitis, which was not originally part of the O’Brien concept, was also discussed in more detail as being an important descriptive term. The original O'Brien concept classification and causes of sporadic VCI [13] are listed for comparison. 


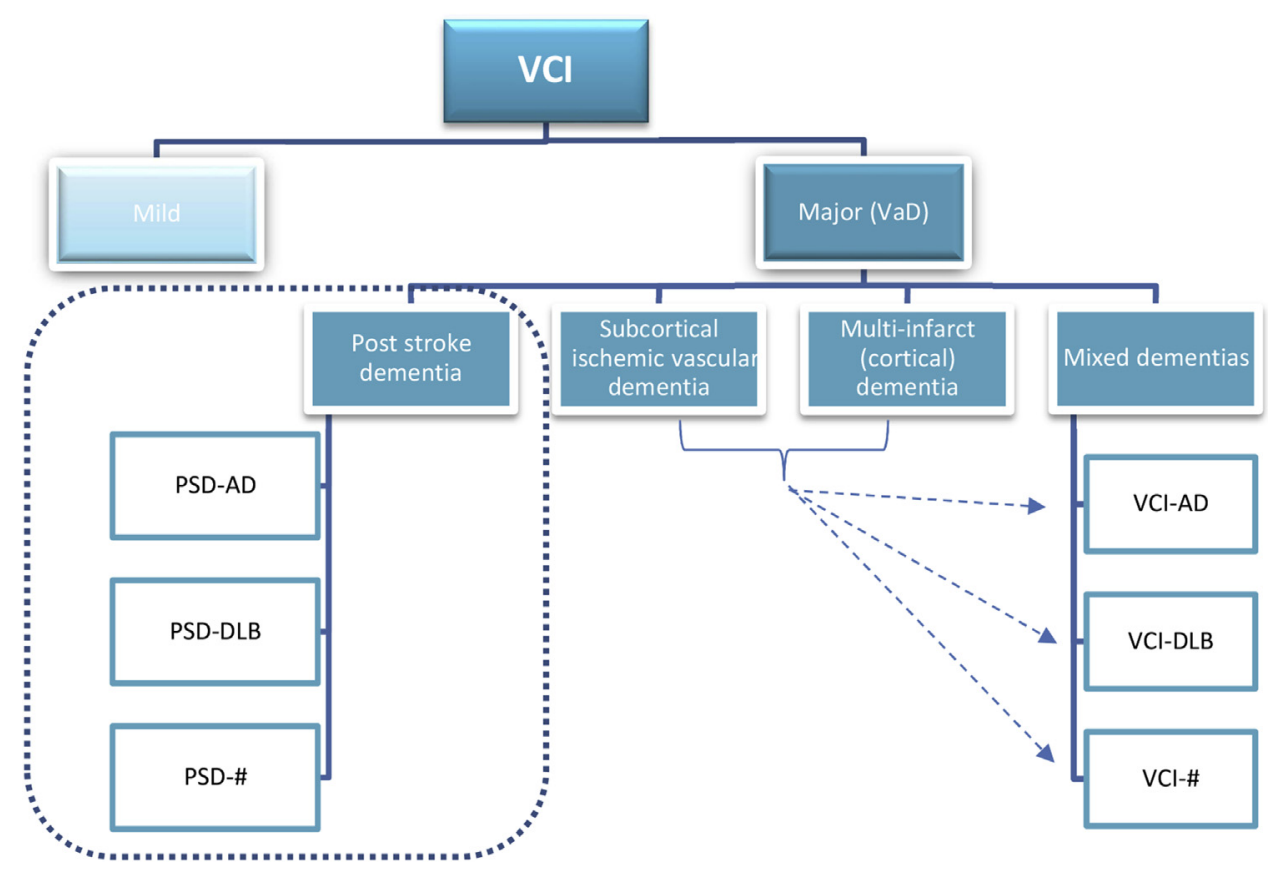

Fig. 1. Revised conceptualization of VCI in VICCCS. Subtypes of VCI are divided according to the level of VCI impairment into mild VCI and major VCI (VaD). Mild VCI is not further subdivided at this time. Major VCI ( VaD) is classified into four main subtypes as depicted. The 6 month temporal basis (denoted by the hashed box) for cognitive decline after stroke differentiates poststroke dementia (PSD) from other forms of major VCI (VaD). PSD and mixed dementias are further delineated if a comorbid neuropathology is present (N.B. AD and dementia with Lewy bodies (DLB) are given as examples, with \# denoting other possible combinations). Subcortical ischemic VaD or multi-infarct (cortical) dementia subtype cases with these specific types of dementia alone, however cases also presenting with any other neurodegenerative pathology would then be categorized as mixed dementias (dashed arrows) according to the comorbidities present. Abbreviations: AD, Alzheimer's disease; VaD, vascular dementia; VCI, vascular cognitive impairment; VICCCS, Vascular Impairment of Cognition Classification Consensus Study.

was agreed (69\%) as a helpful descriptive term of cause (Supplementary Table 2). The resultant VICCCSrecommended subtypes and descriptive terms are presented in Table 2.

\subsubsection{Descriptiveness_clear definitions}

\subsubsection{Mixed dementias}

Mixed dementia and its definition in clinical practice and research were identified as needing elucidation from the earliest rounds, with $97 \%$ of respondents favoring change to the traditional imprecise usage. In the final Delphi round, $95 \%$ of respondents agreed with a proposed solution to the differences in opinion on the term (detailed in Supplementary Material). "Mixed dementias" proposed should serve only as an "umbrella" term for subtypes of major VCI (VaD) under which all phenotypes present would be specified. Patients would be referred to as having for example; VCI-AD, VCI-dementia with Lewy bodies, so forth according to whatever dementia comorbidities presented. A large number of respondents $(81 \%)$ endorsed this approach for both research and clinical applications, and consensus (68\%) was that the order of abbreviations should reflect the relative contributions of the comorbidities, as far as practicable.

\subsubsection{Poststroke dementia}

There was consensus for the term PSD to be used in research $(73 \%)$ and clinical $(86 \%)$ contexts, but no consensus (63\%) around how PSD was previously described, which we had tried to address in later rounds and continued to do in VICCCS diagnosis. Related issues thought necessary to clarify PSD, including evidence of cognitive impairment before stroke and timeframes for the emergence of PSD, are detailed in Supplementary Material. VICCCS consensus (78\%) views on delineation of PSD are detailed in Box 2 and Fig. 1. Of note is the temporal association between cognitive decline and stroke differentiates' PSD from other forms of major VCI ( VaD), that is, cognitive impairment within 6 months of having a stroke would be the determining factor for a diagnosis of PSD.

Consensus proposed definitions for major VCI (VaD) subtypes (PSD, mixed dementias, subcortical ischemic $\mathrm{VaD}$, and multi-infarct dementia) are presented in Box 2.

\section{Discussion}

VICCCS has provided revision and consensus-based elaboration of the construct of VCI in most areas addressed. Lack of consensus in some areas was mainly because of a 
few research data available at the time, for example, the subcategorization of mild forms of VCI. VICCCS showed that although half of the respondents wanted to lessen the overemphasis on memory-impairment in the conceptualization of VCI, two-thirds acknowledged the benefit in the amnestic separation to facilitate alignment with current formats used for $\mathrm{AD}$ and mild cognitive impairment. Thus, subtypes of VCI require more research-based justification.

Definition of more homogeneous groups was supported for major VCI-also important for clinical trial design. Clinical diagnosis of coexisting pathologies remains a challenge. Previous definitions of mixed dementia were not greatly supported in VICCCS, partly because of dissatisfaction with the overemphasis of AD (see Supplementary Material). When the study concluded, a revised concept of the most favored definition (25\% support) has been published for "mixed AD" [30] that does provide separate criteria for coexisting CVD and Lewy body pathology, however does not differentiate these by terminology. VICCCS proposes in mixed dementias and PSD that all phenotypes identified should be specified, depending on whatever dementia-related comorbidities are present, wherein the order of abbreviations reflects the perceived relative contributions. Improvements to the practicalities and accuracy of this would be important aspects of any future operational diagnostic protocols, whereas ongoing research in biomarkers may be helpful. Recent evidence lends weight to this approach, where subcortical VaD can be identified in an outpatient memory clinic setting according to the neuropsychological features and CSF-biochemical markers distinct from those of $\mathrm{AD}$ [38]. Box 3 summarizes this and other areas for future research either proposed or reflected in responses from VICCCS.

VICCCS was conducted between 2010 and 2013 that coincided with the development of DSM-5 [39] and International Society for Vascular Behavioral and Cognitive Disorders (VASCOG) criteria for VCD [36]. VICCCS participants were given the opportunity to provide collective feedback on draft DSM-5 proposals that were made available before its finalization. This was enabled through a tailored survey developed (by O.A.S.) in consultation with P.S. acting on behalf of the DSM-5 Neurocognitive Disorders Work Group and was prompted by their online request for input from the clinical research community into the refinement process. Awareness among VICCCS participants of this request was relatively modest, demonstrating a need for wider advertisement of such consultations in future. VICCCS participants agreed that the mild and major terminologies proposed in DSM-5 were helpful and similarly should be adopted in VICCCS.

In relation to the subsequent published criteria (in 2014) for VCDs, VICCCS had previously explored but was not supportive of this concept and the use of this term VCD $[11,17]$. However, the VASCOG criteria are also reported to be aligned with DSM-5 [36].

\subsection{Considerations of the Delphi process on VICCCS outcomes}

A key principle of the Delphi method is that decisions from a structured specialist group of individuals are more accurate. The use of online surveys in VICCCS, to avoid scheduling constraints of a physical meeting, facilitated the inclusion of an unprecedented large number of international participants who have enriched discussions. The anonymity offered by Delphi reduced the potential for any individuals to dominate direction of discussions. Furthermore, in combination with the repeated group feedback, the anonymity allowed contemplation, review of initial judgments, and scope for participants to freely change opinions, all of which promoted the generation of consensus $[34,40]$. The use of specific published articles helped to focus the discussion points and in some cases, increased awareness of previous studies, aiding more-informed decision making. After the initial rounds, structured, mostly closed questions were mainly used to ensure continued focus when some feedback was possible, in the primary discussion of topics. This sometimes extended the duration of the study and

Box 3

Potential areas for future research as proposed directly or identified from responses from the VICCCS.

1. Evidence-based studies to support further subdivision of mild VCI.

2. Develop a more systematic stepwise approach toward subtyping of patients based on new VICCCS proposed categories of location, etiology, domains (affected), and severity.

3. Investigation of factors that determine immediate or delayed onset of VCI in PSD patients.

4. Investigation of factors (e.g., time to onset, biomarkers, cognitive parameters) that may better delineate comorbidity of PSD with other causes of VCI or nonvascular dementias.

5. Further elucidation to improve phenotyping of relative contribution of the co-occurring pathology, for example, ADVCI or VCI-AD in mixed dementias, or other neurodegenerative diseases (e.g., Parkinson's disease) or psychiatric disorders that copresent with CVD.

6. Further exploration of the utility and validity of the traditional term multi-infarct dementia as either a specific-subtype of major $\mathrm{VCI}(\mathrm{VaD})$ or as a complementary descriptive term alongside the newly proposed subtypes of major VCI $(\mathrm{VaD})$ 
complexity of the arguments, such as the discussion of mixed dementias and PSD. Yet the extended debate was useful but increased risk of participant attrition, and variation in respondent numbers in each round did variably impact on the relative contribution of each respondent toward consensus. However, most topics were dealt with over multiple rounds giving many opportunities to confirm the consensus view. The maintenance of a high number of participants throughout the study provides assurance that a consensus concept of VCI has been realized, although by definition the consensus was based on a majority view.

\section{Conclusions}

VICCCS presents a new consensus-based set of guidelines supported by a large international pool of researchers. These guidelines have drawn on, expanded, and refined previous efforts to improve and clarify the conceptualization of VCI. It is anticipated that VICCCS guidelines will be widely adopted in the community to increase the levels of consistency and standardization in undertaking VCI research. This should significantly enhance the interpretation and comparison of findings across studies and support the likelihood of more large-scale collaborative research that will be vital to help overcome historical limitations posed by the prevalence of VCI.

\section{Acknowledgments}

This work was supported by a project grant (Ref117) from the Alzheimer's Society (UK).

Author contributions: O.A.S. was the study coordinator, analyzed the data, formulated the questionnaires, and wrote the manuscript. P.G.K. was Chief investigator, conceived and designed the study, obtained the necessary funding, reviewed each round data, formulated the questionnaires, and wrote the manuscript. Y.B.-S., A.P.P., and S.L. were coinvestigators and members of the Steering Group. Other listed authors were members of the Steering Group who reviewed the content of the pilot questionnaires, draft, and final manuscript and were participants in the study. Authors listed under the banner of Vascular Impairment of Cognition Classification Consensus Study groups contributed to data gathering in multiple survey rounds and approved the final submitted version of the article.

VICCCS group: Argentina: F.E. Taragano, CONICET National Research Council and CEMIC University Hospital. Australia: J. Kril, University of Sydney. Austria: M. Cavalieri, Medical University of Graz; K.A. Jellinger, Institute of Clinical Neurobiology; G.G. Kovacs, Medical University of Vienna. Belgium: S. Engelborghs, University of Antwerp; C. Lafosse, RevArte Rehabilitation Hospital and Catholic University of Leuven. Brazil: P.H. Bertolucci, Universidade Federal de Sao Paulo; S. Brucki, University of Sao Paulo; P. Caramelli, Universidade Federal de Minas Gerais; T. C de Toledo Ferraz Alves, Department of Psychiatry of São Paulo
Medical School. Canada: C. Bocti, Université de Sherbrooke; T. Fulop, Université de Sherbrooke; D.B. Hogan, University of Calgary; G.R. Hsiung, University of British Columbia; A. Kirk, University of Saskatchewan; L. Leach, Glendon College, York University; A. Robillard, Hopital Maisonneuve; D.J. Sahlas, McMaster University. People's Republic of China: Q. Guo, Huashan Hospital, Fudan University; J. Tian, Dongzhimen Hospital, Beijing University of Chinese Medicine. Finland: L. Hokkanen, University of Helsinki; H. Jokinen, Helsinki University Hospital. France: S. Benisty, Institution Nationale des Invalides; V. Deramecourt, Lille University Hospital; J. Hauw, APHP, Pitié-Salpêtrière Hospital, and Pierre et Marie-Curie University; H. Lenoir, Broca Hospital-HUPCAPHP and Paris-Descartes 5 University. Greece: M. Tsatali, Greek Alzheimer Association; M. Tsolaki, Aristotle University of Thessaloniki. India: U. Sundar, Lokmanya Tilak Municipal Medical College and Hospital, Sion. Ireland: R.F. Coen, Mercer's Institute for Research on Ageing, St. James's Hospital Dublin. Israel: A.D. Korczyn, Tel Aviv University. Italy: M. Altieri, Sapienza Università di Roma; M. Baldereschi, Italian National Research Council; C. Caltagirone, Rome University of Tor Vergata and Santa Lucia IRCCS Foundation Rome; G. Caravaglios, Azienda Ospedaliera Cannizzaro, Catania; A. Di Carlo, Institute of Neuroscience, Italian National Research Council; V. DI Piero, Sapienza University; G. Gainotti, Catholic University; S. Galluzzi, IRCCS Istituto Centro San Giovanni di Dio-Fatebenefratelli; G. Logroscino, University of Bari; P. Mecocci, University of Perugia; D.V. Moretti, IRCCS Istituto Centro San Giovanni di DioFatebenefratelli; A. Padovani, Università degli Studi di Brescia. Japan: T. Fukui, Kawasaki Memorial Hospital; M. Ihara, Kyoto University; T. Mizuno, Kyoto Prefectural University of Medicine. Republic of Korea: S.Y. Kim, Seoul National University Bundang Hospital. Nigeria: R. Akinyemi, University of Ibadan and Newcastle University UK; O. Baiyewu, University of Ibadan; A. Ogunniyi, University of Ibadan. Poland: A. Szczudlik, Jagiellonian University Medical College. Portugal: A.J. Bastos-Leite, University of Porto; H. Firmino, Coimbra University Hospital; J. Massano, University of Porto and Hospital Pedro Hispano/ULS Matosinhos; A. Verdelho, University of Lisbon, Hospital de Santa Maria. Russia: L.S. Kruglov, St. Petersburg University and St. Petersburg Psychoneurological Research Institute. Singapore: M.K. Ikram, National University of Singapore and Erasmus Medical Centre, Rotterdam; N. Kandiah, National Neuroscience Institute. Spain: E. Arana, Fundación IVO; J. Barroso-Ribal, University of La Laguna; T. Calatayud, Hospital Universitario Central de Asturias; A.J. Cruz-Jentoft, Hospital Universitario Ramón y Cajal Madrid; S. López-Pousa, Institut Català de la Salut, Girona and Institut d'Assistència Sanitària, Catalonia; P. Martinez-Lage, Fundacion CITA Alzheimer; M. Mataro, University of Barcelona. Sweden: A. Börjesson-Hanson, Neuroscience and Physiology, Sahlgrenska Academy at the University of Gothenburg; E. Englund, Lund University; E.J. Laukka, Karolinska Institutet; C. Qiu, Karolinska Institutet; M. Viitanen, Karolinska Institutet, University of Turku. The 
Netherlands: G.J. Biessels, University Medical Center Utrecht; F.-E. de Leeuw, Radboud University Nijmegen Medical Centre and Donders Institute for Brain Cognition and Behaviour; T. den Heijer, Sint Franciscus Gasthuis; L.G Exalto, UMCU; L.J. Kappelle, University Medical Centre Utrecht; N.D. Prins, V.U. University Amsterdam; E. Richard, University of Amsterdam and Radboud University, Nijmegen; B. Schmand, University of Amsterdam; E. van den Berg, University Medical Center Utrecht; W.M. van der Flier, VU University Medical Center. Turkey: B. Bilgic, Istanbul University. United Kingdom: L.M. Allan, Newcastle University; J. Archer, Mid-Yorkshire NHS Trust; J. Attems, Newcastle University; A. Bayer, Cardiff University; D. Blackburn, University of Sheffield; C. Brayne, University of Cambridge; R. Bullock, Kingshill Research Centre; P.J. Connelly, University of Dundee, Murray Royal Hospital, Perth; A. Farrant, NHS; M. Fish, Musgrove Park Hospital; K. Harkness, Sheffield Teaching Hospital Foundation Trust; P.G. Ince, University of Sheffield; P. Langhorne, Glasgow University; J. Mann, The Research Institute for the Care of Older People; F.E. Matthews, MRC Biostatistics Unit; P. Mayer, Institute of ageing West Midlands; S.T. Pendlebury, Stroke Prevention Research Unit and NIHR Biomedical Research Centre; R. Perneczky, Imperial College London; R. Peters, Imperial; D. Smithard, King's College Hospital, London and University of Kent; B.C Stephan, Newcastle University; J.E Swartz, Bracket Global; S. Todd, Western Health and Social Care Trust; D.J. Werring, Stroke Research Centre, UCL Institute of Neurology; S.N. Wijayasiri, Bedford Hospital; G. Wilcock, University of Oxford; G. Zamboni, Nuffield Department of Clinical Neurosciences (NDCN), University of Oxford. United Sates of America: R. $\mathrm{Au}$, Boston University; S. Borson, University of Washington School of Medicine; A. Bozoki, Michigan State University; J.N. Browndyke, Duke University Medical Center; M.M. Corrada, University of California, Irvine; P.K. Crane, University of Washington; B.S. Diniz, University of Texas Health Science Center at Houston; L. Etcher, L Etcher, Spring Arbor University; H. Fillit, The Alzheimer's Drug Discovery Foundation; S.M. Greenberg, Massachusetts General Hospital and Harvard Medical School; L.T. Grinberg, University of California San Francisco and University of Sao Paulo Medical School; S.W. Hurt, Weill Cornell Medical College; M. Lamar, University of Illinois at Chicago and Institute of Psychiatry, King's College London UK; M. Mielke, Mayo Clinic; B.R. Ott, Brown University; G. Perry, University of Texas at San Antonio; W.J Powers, University of North Carolina; C RamosEstebanez, Case Western Reserve University; B. Reed, University of California, Davis; R.O. Roberts, Mayo Clinic; J.R. Romero, Boston University; A.J. Saykin, Indiana University; S. Seshadri, Boston University; L. Silbert, Oregon Health \& Science University; Y. Stern, Columbia University; C. Zarow, University of Southern California.

Declaration of interests: Prof. Ford reports personal fees from Pfizer, Athersys, AstraZeneca, Lundbeck, Cerevast, and Daiichi Sankyo and grants and personal fees from Boehringer Ingelheim, outside the submitted work. Prof. O'Brien reports personal fees from GE Healthcare, TauRx, and Cytox and grants and personal fees from Avid/Lilly, outside the submitted work. Prof. Skoog reports personal fees and other from Takeda, outside the submitted work. Outside the submitted work, Prof. Black reports institutional grants from Pfizer, GE Healthcare, Eli Lilly, Elan/Transition Therapeutics, Roche, and Cognoptix and personal fees from Pfizer, GE Healthcare, Eli Lilly, Eisai, Boehringer Ingelheim, and Novartis.

\section{Supplementary data}

Supplementary data related to this article can be found at http://dx.doi.org/10.1016/j.jalz.2016.10.007.

\section{RESEARCH IN CONTEXT}

1. Systematic review: Capturing the complexity involved in vascular cognitive impairment (VCI) has given rise to various terms such as multi-infarct dementia, vascular dementia, subcortical (ischemic) vascular dementia, VCI, and vascular cognitive disorders. These terms were used as search-terms in Pubmed for relevant published reports (by August 2010). The authors list identified potential study participants and a Steering Group. Twelve concept articles and 15 articles proposing diagnostic criteria were used in the Delphi study.

2. Interpretation: This process resulted in the formation of new consensus-based guidelines for VCI that have drawn on and refined previous initiatives. High levels of participation by the research community should ensure these guidelines will be more widely endorsed.

3. Future directions: Key research priorities include evidence-based studies to (1) determine appropriate subdivision of mild VCI and (2) improve phenotyping of the co-occurring pathology in mixed dementias, and other neurodegenerative diseases or psychiatric disorders that copresent with cerebrovascular disease.

\section{References}

[1] Snowdon DA, Greiner LH, Mortimer JA, Riley KP, Greiner PA, Markesbery WR. Brain infarction and the clinical expression of Alzheimer disease. The Nun Study. JAMA 1997;277:813-7.

[2] Launer LJ, Hughes TM, White LR. Microinfarcts, brain atrophy, and cognitive function: the Honolulu Asia Aging Study Autopsy Study. Ann Neurol 2011;70:774-80. 
[3] Vermeer SE, Prins ND, den Heijer T, Hofman A, Koudstaal PJ, Breteler MM. Silent brain infarcts and the risk of dementia and cognitive decline. N Engl J Med 2003;348:1215-22.

[4] Smallwood A, Oulhaj A, Joachim C, Christie S, Sloan C, Smith AD, et al. Cerebral subcortical small vessel disease and its relation to cognition in elderly subjects: a pathological study in the Oxford Project to Investigate Memory and Ageing (OPTIMA) cohort. Neuropathol Appl Neurobiol 2012;38:337-43.

[5] Kester MI, Scheltens P. Dementia: the bare essentials. Pract Neurol 2009;9:241-51.

[6] Yoshitake T, Kiyohara Y, Kato I, Ohmura T, Iwamoto H, Nakayama K, et al. Incidence and risk-factors of vascular dementia and Alzheimersdisease in a defined elderly Japanese population — the Hisayama study. Neurology 1995;45:1161-8.

[7] Ott A, Stolk RP, Hofman A, vanHarskamp F, Grobbee DE, Breteler MM. Association of diabetes mellitus and dementia: the Rotterdam study. Diabetologia 1996;39:1392-7.

[8] Dwyer R, Skrobot OA, Dwyer J, Munafo M, Kehoe PG. Using Alzgene-like approaches to investigate susceptibility genes for vascular cognitive impairment. J Alzheimers Dis 2013;34:145-54.

[9] Hachinski VC, Lassen NA, Marshall J. Multi-infarct dementia. A cause of mental deterioration in the elderly. Lancet 1974;2:207-10.

[10] Kalaria RN, Kenny RA, Ballard CG, Perry R, Ince P, Polvikoski T. Towards defining the neuropathological substrates of vascular dementia. J Neurol Sci 2004;226:75-80.

[11] Sachdev P. Vascular cognitive disorder. Int J Geriatr Psychiatry 1999; 14:402-3.

[12] Rockwood K, Macknight C, Wentzel C, Black S, Bouchard R, Gauthier S, et al. The diagnosis of "mixed" dementia in the Consortium for the Investigation of Vascular Impairment of Cognition (CIVIC). Ann N Y Acad Sci 2000;903:522-8.

[13] O’Brien JT, Erkinjuntti T, Reisberg B, Roman G, Sawada T, Pantoni L, et al. Vascular cognitive impairment. Lancet Neurol 2003;2:89-98.

[14] Zhao QL, Zhou Y, Wang YL, Dong KH, Wang YJ. A new diagnostic algorithm for vascular cognitive impairment: the proposed criteria and evaluation of its reliability and validity. Chin Med J (Engl) 2010; 123:311-9.

[15] Cao X, Guo Q, Zhao Q, Jin L, Fu J, Hong Z. The neuropsychological characteristics and regional cerebral blood flow of vascular cognitive impairment-no dementia. Int J Geriatr Psychiatry 2010;25:1168-76.

[16] Hachinski VC, Bowler JV. Vascular dementia. Neurology 1993; 43:2159-60. author reply 2160-1.

[17] Roman GC, Sachdev P, Royall DR, Bullock RA, Orgogozo JM, LopezPousa S, et al. Vascular cognitive disorder: a new diagnostic category updating vascular cognitive impairment and vascular dementia. J Neurol Sci 2004;226:81-7.

[18] Rockwood K, Wentzel C, Hachinski V, Hogan DB, MacKnight C, McDowell I. Prevalence and outcomes of vascular cognitive impairment. Vascular Cognitive Impairment Investigators of the Canadian Study of Health and Aging. Neurology 2000;54:447-51.

[19] Erkinjuntti T, Inzitari D, Pantoni L, Wallin A, Scheltens P, Rockwood K, et al. Research criteria for subcortical vascular dementia in clinical trials. J Neural Transm Suppl 2000;59:23-30.

[20] Chui HC, Victoroff JI, Margolin D, Jagust W, Shankle R, Katzman R. Criteria for the diagnosis of ischemic vascular dementia proposed by the State of California Alzheimer's Disease Diagnostic and Treatment Centers. Neurology 1992;42(3 Pt 1):473-80.

[21] Roman GC, Tatemichi TK, Erkinjuntti T, Cummings JL, Masdeu JC, Garcia JH, et al. Vascular dementia—diagnostic-criteria for research studies-report of the NINDS-AIREN International Workshop. Neurology 1993;43:250-60.

[22] Chui HC, Mack W, Jackson JE, Mungas D, Reed BR, Tinklenberg J, et al. Clinical criteria for the diagnosis of vascular dementia: a multicenter study of comparability and interrater reliability. Arch Neurol 2000;57:191-6.

[23] Wiederkehr S, Simard M, Fortin C, van Reekum R. Validity of the clinical diagnostic criteria for vascular dementia: a critical review. Part II. J Neuropsychiatry Clin Neurosci 2008;20:162-77.

[24] Roman GC. Vascular dementia may be the most common form of dementia in the elderly. J Neurol Sci 2002;203:7-10.

[25] Satizabal CL, Beiser AS, Chouraki V, Chene G, Dufouil C, Seshadri S Incidence of dementia over three decades in the Framingham Heart Study. N Engl J Med 2016;374:523-32.

[26] Toledo JB, Arnold SE, Raible K, Brettschneider J, Xie SX, Grossman M, et al. Contribution of cerebrovascular disease in autopsy confirmed neurodegenerative disease cases in the National Alzheimer's Coordinating Centre. Brain 2013;136(Pt 9):2697-706.

[27] Attems J, Jellinger KA. The overlap between vascular disease and Alzheimer's disease-lessons from pathology. BMC Med 2014;12:206.

[28] Korczyn AD. Mixed dementia-the most common cause of dementia. Ann N Y Acad Sci 2002;977:129-34.

[29] Andrieu S, Coley N, Lovestone S, Aisen PS, Vellas B. Prevention of sporadic Alzheimer's disease: lessons learned from clinical trials and future directions. Lancet Neurol 2015;14:926-44.

[30] Dubois B, Feldman HH, Jacova C, Hampel H, Molinuevo JL, Blennow K, et al. Advancing research diagnostic criteria for Alzheimer's disease: the IWG-2 criteria. Lancet Neurol 2014;13:614-29.

[31] Brayne C, Davis D. Making Alzheimer's and dementia research fit for populations. Lancet 2012;380:1441-3.

[32] Grinberg LT, Heinsen H. Toward a pathological definition of vascular dementia. J Neurol Sci 2010;299:136-8.

[33] Hachinski V, Iadecola C, Petersen RC, Breteler MM, Nyenhuis DL, Black SE, et al. National Institute of Neurological Disorders and Stroke-Canadian Stroke Network vascular cognitive impairment harmonization standards. Stroke 2006;37:2220-41.

[34] Dalkey N, Helmer O. An experimental application of the Delphi method to the use of experts. Manag Sci 1963;9:458-67.

[35] Alexandrov AV, Pullicino PM, Meslin EM, Norris JW. Agreement on disease-specific criteria for do-not-resuscitate orders in acute stroke. Members of the Canadian and Western New York Stroke Consortiums. Stroke 1996;27:232-7.

[36] Sachdev P, Kalaria R, O'Brien J, Skoog I, Alladi S, Black SE, et al.International Society for Vascular, Behavioral, and Cognitive Disorders. Diagnostic criteria for vascular cognitive disorders: a VASCOG statement. Alzheimer Dis Assoc Disord 2014;28:206-18.

[37] Ganguli M, Blacker D, Blazer DG, Grant I, Jeste DV, Paulsen JS, et al. Classification of neurocognitive disorders in DSM-5: a work in progress. Am J Geriatr Psychiatry 2011;19:205-10.

[38] Wallin A, Nordlund A, Jonsson M, Blennow K, Zetterberg H, Ohrfelt A, et al. Alzheimer's disease-subcortical vascular disease spectrum in a hospital-based setting: overview of results from the Gothenburg MCI and dementia studies. J Cereb Blood Flow Metab 2016; 36:95-113.

[39] American Psychiatric Association. Diagnostic and statistical manual of mental disorders. 5th ed. Arlington: American Psychiatric Publishing; 2013.

[40] Delbecq AL, Van de Ven AH, Gustafson DH. Group techniques for program planning. Glenview, IL: Scott, Foresman, and Co; 1975. 